\title{
Research and Design of the Coal Mine Safety Supervision System Based on IOT
}

\author{
Liwang $\mathrm{Zhu}^{1}$ \\ School of Computer Science and \\ Engineering Hunan University of \\ Science and Technology Xiangtan, \\ P.R.China divid_zhu@163.com
}

\author{
Shaobo Zhang $^{2}$ \\ School of Computer Science and \\ Engineering Hunan University of \\ Science and Technology Xiangtan, \\ P.R.China27603599@qq.com
}

\author{
Chengzhang $\mathrm{Zhu}^{3}$ \\ College of Computer National \\ University of Defense Technology \\ Changsha, P.R.China \\ kevin.zhu.china@gmail.com
}

\begin{abstract}
This paper studied the coal mine safety supervision technology systematically, and proposed an overall structure of an integrated safety supervision system with environment supervision, mine equipment monitoring and person / vehicle location management functions. Then, it intensively analyzed the functional requirements of the system hardcore supervision substation; and carried out the HW\&SW design and development of the supervision substation device which includes CAN bus interface, ZigBee coordinator and various function modules. Finally, by building up a simulation test environment, the performance of the system structure and main functions of the substation device were validated.
\end{abstract}

Keyword-Mine Safety; Internet of Things (IOT); CAN Bus; ZigBee Protocol; Supervision Substation

\section{INTRODUCTION}

Safety is the eternal theme of the coal industry. Due to complex geological circumstance and poor production conditions in coal mines, most of the previous monitoring systems are based on the wired network architecture. However, this kind of architecture also brings some problems: underground deployment of these systems is difficult; and because the equipment based on wired communication is poor in mobility and scalability, it also is difficult to fully monitor all areas of mines. On the other hand, most of the previous systems only cover the monitoring of environmental parameter \& mine equipment operating status, but person / vehicle locating and management system which could put forward instructions for rescue and evacuation of persons after the accident needs to be re-invested and built separately, moreover, because of lacking information exchange, it is difficult to ensure the effectiveness of relief efforts.

\section{THE OVERALL RESEARCH ON SUPERVISION SYSTEM}

\section{A. The overall study and analysis}

The main goal of mine safety supervision system is to effectively protect the safety of mine workers, as well as the safety of mining production [1]. Therefore, an integrated monitoring system for the underground mine should cover the monitoring of environmental parameter \& mine equipment operating status, so that it is able to provide decision-making basis for ensuring safety in production and avoiding accidents disaster; and after analyzing and controlling it should be able to prevent expanding of accident; finally, it should have an emergency rescue handling capability by tracking the location of the staff and vehicles.

Special environment of the underground coal mine always brings inconvenience and difficulties in deployment, installation \& maintenance. Fortunately, with the electronics and sensor technology progressing, nowadays, monitoring of various environmental parameters may adopt wireless sensor technology solutions [2]; in the way of communication, because underground mine is not conducive to long-distance wireless transmission, the backbone communication-network may take the wired digital communication mode in roadways of the mine and the main mining areas. Corresponding to perception layer, network layer and application layer of internet of things (IOT), the mine safety supervision system may be designed into the following three-tier network architecture. [3]:

(1) The wireless sensor network (WSN) deployed in branch roadways and local regions of the mine acts as the perception layer.

(2) The wired backbone digital communication network placed in roadways and the main mining areas acts as the network layer.

(3) The ground supervision host and the remote users act as the application layer.

Figure 1 shows the network architecture of the safety supervision system based on IOT and WSN technology.

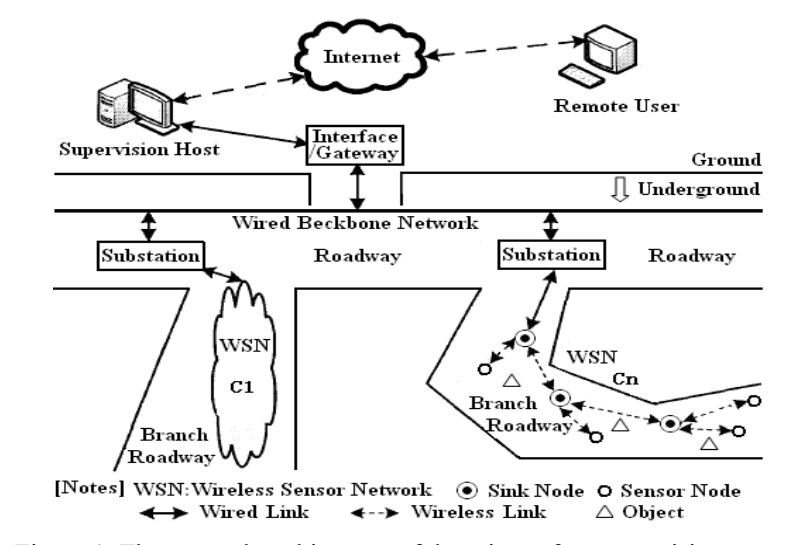

Figure 1. The network architecture of the mine safety supervision system

\section{$B$. The construction of the supervision system}

In accordance with the relevant national industry standards, a coal mine safety monitoring and surveillance system should be consisted of monitoring host, monitoring 
substation, methane power breaker, sensor (transducer), transmission interface, and etc.

According to the system network architecture, for the construction of the backbone communication network, the CAN bus communication technology may be used. CAN bus protocol is set up by the German BOSCH company, which is the first international standard of field-bus specifications and widely applied to the transportation, defense, engineering, industrial, security and many other areas [ 4 ] . It has great advantages in the communication distance \& transmission rate [5]: the maximum transmission distance reaches $10 \mathrm{~km}$, and the maximum communication rate is up to $1 \mathrm{Mbs}$. However, for the wireless sensor network (WSN), ZigBee technology may be adopted. ZigBee (IEEE802.15.4) is an emerging short-range wireless transmission protocol standards developed in recent years, compared with several other short-range wireless transmission protocols such as Bluetooth, Wi-Fi, IrDA, it holds the following advantages [6].

(1) Low power consumption. The duty cycle of ZigBee node equipments is short, and messaging power also is low.

(2) Reliable transmission and immunity to interference. Adopting DSSS communication mode, compared to the RFID broadband and $433-915 \mathrm{MHz}$ FSK communication mode, its anti-interference capability is stronger.

(3) Low product cost. Because the design of ZigBee protocol stack is concise, so its $\mathrm{R} \& \mathrm{D}$ costs are relatively low and the software also is relatively simple.

(4) High speed and long distance. 2.4GHz ZigBee protocol physical layer, its transmission rate reaches $250 \mathrm{kbps}$; the transmission distance is $30-70 \mathrm{~m}$, and it may exceed $100 \mathrm{~m}$ by amplifying communication signal.

ZigBee communication protocol defined three kinds of roles node as follows.

(1) ZigBee Coordinator that is responsible for network configuring and creating. It is the central node of the network and the full-function device (FFD);

(2) ZigBee Router which is responsible for message forwarding and routing, it also is the FFD device;

(3) ZigBee End Device which performs a specific function, it is also known as the sensor node, and may be the full-function device or the reduced function device (RFD).

The construction of supervision system shows in Fig. 2.

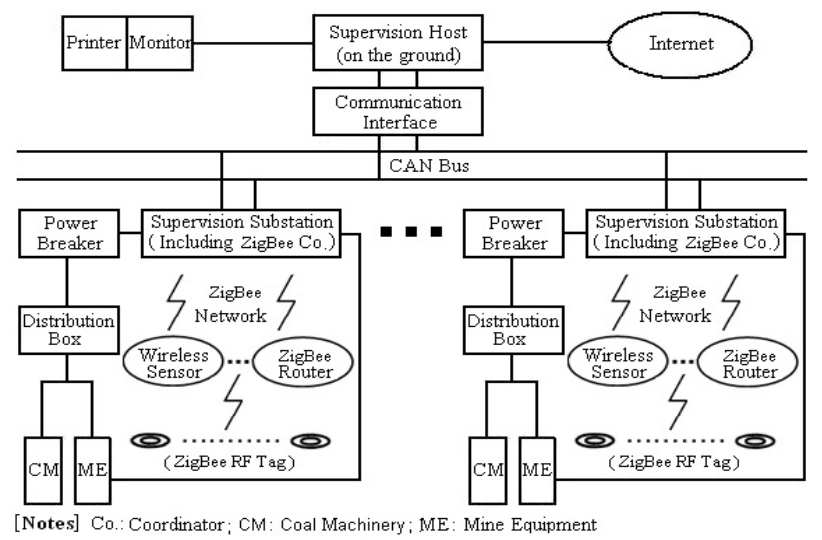

Figure 2. The construction of the supervision system
System is composed of the ground supervision host, the supervision substation (including ZigBee coordinator), wireless sensor node, router node, ZigBee RF tags, as well as power switch controllers and other ancillary equipments. Communication interface may be CAN-PCI, CAN-USB, or CAN-Ethernet interface card.

ZigBee wireless sensors and RF tags are ZigBee end devices. Types of sensors may include methane concentration, $\mathrm{CO}$ concentration, temperature, humidity, dust concentration, velocity, water pressure, displacement, stress, and negative pressure. The router node is also called the reference node, the position of which is manually configured. It is responsible for information collecting of sensor nodes, forwarding data to other network nodes, and obtaining person / vehicle coordinates combining with mobile nodes. ZigBee electronic tag is the mobile node, and the position information of these tags is transmitted to the ZigBee coordinator through router nodes in order to achieve the location and tracking of personnel/vehicle underground.

\section{DESIGN OF THE SUPERVISION SUBSTATION}

\section{A. Realization of the ZigBee network coordinator}

The supervision substation (SST) is the core of the entire mine safety supervision system. According to the overall design frame of the system, the substation device should include hardware for implementing ZigBee network coordinator functions.

The universal module structure of ZigBee network nodes (coordinator, router and sensor) is shown in Figure 3, where some modules may be selected by the actual needs.

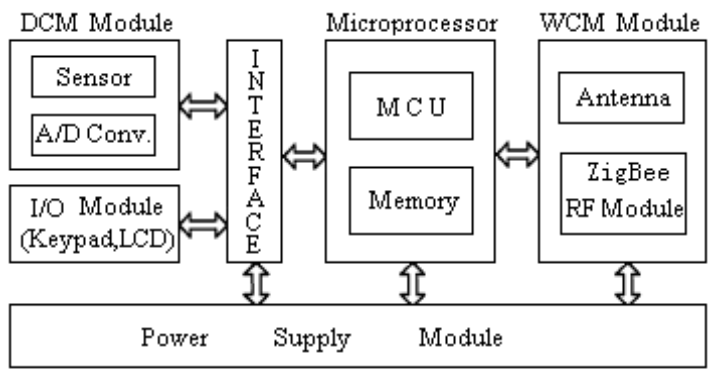

[Notes] DCM: Data Collection Module; RF: Radio Frequency WCM: The Wireless Communication Module

Figure 3. Block diagram of ZigBee nodes

According to the structure of ZigBee nodes and functional requirements of the SST device, apart from the wireless communication module, the other functional modules can both be used in the SST device and the ZigBee coordinator; combining the common modules and the wireless communication module it is fully able to construct an integrated supervision substation device which includes ZigBee coordinator.

\section{B. The overall design of the SST}

According to the overall design and function requirements of the SST, SST hardware may be composed of 
the microprocessor (MCU), network communication interfaces, mine equipment monitoring circuit, interactive feature circuit and power supply circuit . The configuration of the supervision substation is shown in Figure 4.

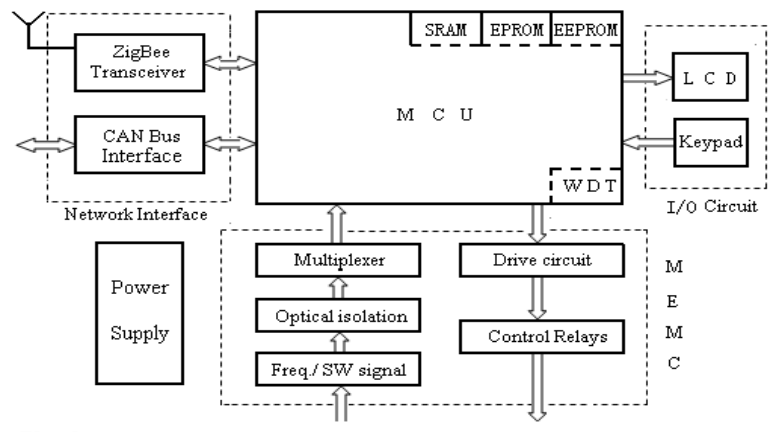

[Notes] Freq//SW : Frequency/Switch; MEMC: Mine Equipment Monitoring Circuit

Figure 4.The configuration of the supervision substation

ZigBee transceiver is the wireless communication interface which used to obtain environmental parameters and personnel / vehicle location information collected by the wireless sensor network; CAN bus interface is used to upload these information and mine equipment statuses to the ground supervision host, or receive instructions from the ground supervision host; the mine equipment monitoring circuit is responsible for equipment status scan and control; Keypad and LCD is responsible for input and display.

\section{Design of main circuit units}

\section{1) CAN bus communication interface}

The CAN bus acts as underground communication backbone network, which is a bridge between the supervision host and substations. CAN bus interface may be constructed by CAN controller SJA1000, CAN transceiver PCA82C250 and optical isolation chip 6N137. Independent CAN controller SJA1000 is responsible for initial setting and transferring messages between microprocessor (MCU) and PCA82C250. CAN transceiver PCA $82 \mathrm{C} 250$ is used to establish the physical layer of CAN bus protocol. CAN bus interface circuit structure is shown in Figure 5.

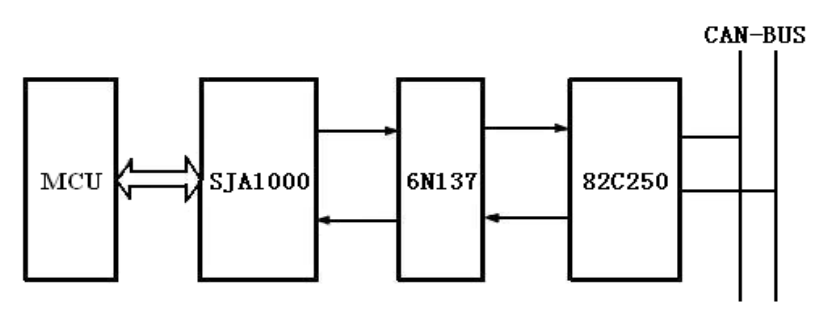

Figure 5. CAN bus interface circuit structure

2) ZigBee network wireless communication interface

The single-chip ZigBee transceiver ZIC2410 is a lowcost high-performance networking solutions for the IEEE 802.15.4 $2.4 \mathrm{GHz}$ wireless network provided by CEL company, which integrated a ZigBee wireless RF transceiver and an embedded 8051CPU nuclear.
Linking UART of the MCU with UART of the ZM2410, assisted by a small number of peripheral components, the supervision substation will possess the ZigBee wireless communication function. On the other hand, downloading coordinator function program into its flash with ISP mode, the ZM2410 module will become a ZigBee coordinator node. Adopting microprocessor STC15F2K60, the connection circuit is shown in Figure 6.

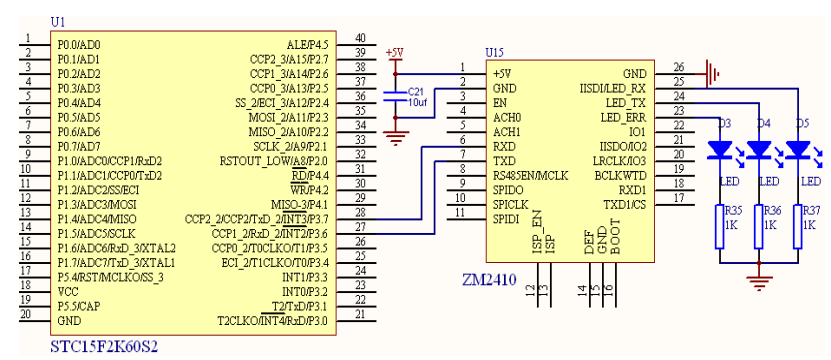

Figure 6. Connection between MCU and ZigBee module

\section{3) Equipment status monitoring circuit}

In order to ensure the normal operation of underground mine equipments, the substation device should include status scanning circuit and relay drive circuit. Operational status of equipment is generally represented by the frequency/switch signal, so these analog input signals should be processed.

\section{DESIGN OF THE MAIN SOFTWARE PROGRAMS}

\section{A. CAN bus communication program}

CAN communication program is responsible for the initialization of CAN bus controller SJA1000, as well as the reception $\&$ transmission of message packets

The reception of message packet: after receiving the data packet, controller SJA1000 will put them into the FIFO reception buffer through the acceptance filter, and then generate a reception interrupt, also set a flag in status register. Microprocessor will responses to this interrupt event, then read and write the data bytes into its own RAM memory, finally releases the reception buffer. The main program codes for the reception of data packets are as follows:

void PeliCANReceive(void) interrupt 0

// Reception function, receiving data packet

$/ /$ and releasing the data reception buffer UINT8 i;

$\{$ If $($ SR\&RBS $)==1)$

// Does reception buffer have data?

\{ CanReceive. Cmd $=$ Data[0];

// Extracting the first byte (control command code)

for $(\mathrm{i}=0 ; \mathrm{i}<7 ; \mathrm{i}++)$

CanReceive. $\operatorname{Arg}[\mathrm{i}]=\operatorname{Data}[\mathrm{i}+1]$;

// Receiving data packet (the remaining 7 bytes)

$\mathrm{CMR}=0 \mathrm{x} 04$;

// Releasing the data reception buffer

\} 


\section{B. ZigBee coordinator functional programming}

After ZigBee networking program is downloaded to the flash memory of the ZIC2410 chip, it will become a ZigBee coordinator node. As a bridge between the upper computer (MCU of the substation device) and the ZigBee wireless network, on the one hand, the coordinator receives data acquired by the ZigBee network, and then uploads these data to the MCU of the substation device; on the other hand, MCU of the substation device also may issue commands to the coordinator, such as "Start capture" command, then coordinator will send data-request to the ZigBee network [7] Additionally, as the first node of a net, coordinator will be responsible for parameters configuration of the network. The two steps of network-establishing are: network initialization, node joining [8]. The process flow shows in Fig.7.

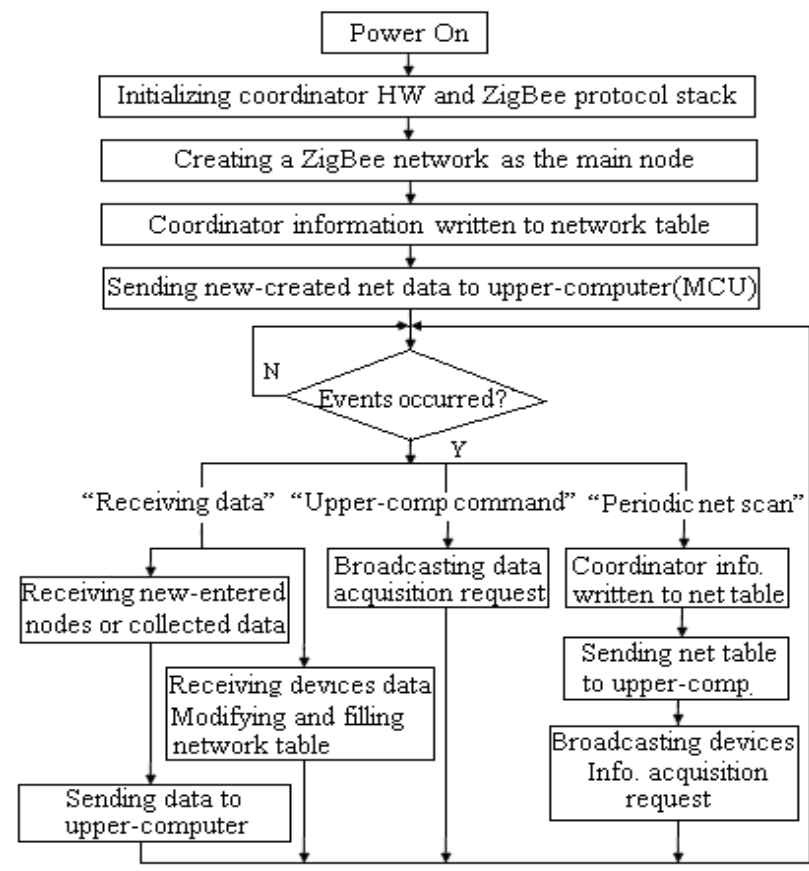

Figure 7. Process flow of the coordinator program

\section{The main program of the substation}

The main tasks of the supervision substation are: performing initialization of the system; issuing commands to the ZigBee coordinator through serial port; executing mine equipment control commands issued by the ground supervision host via the CAN bus; periodically scanning mine equipment status; monitoring mine equipments; receiving environment parameters and staff location information; uploading these information to the ground supervision host. The process flow shows in Figure 8.

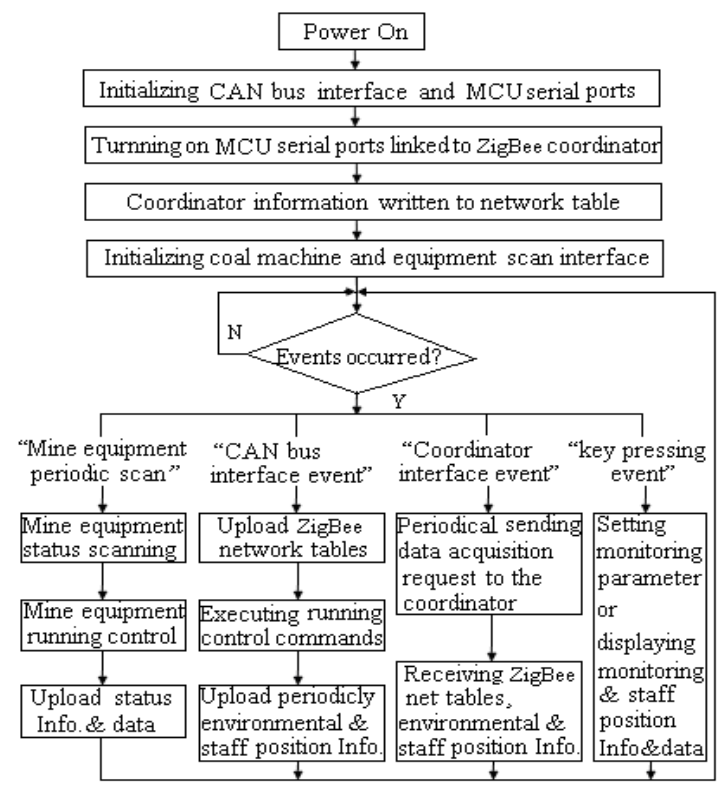

Figure 8. Processing flow of the substation main program

\section{CONCLUSION}

This paper proposed an overall frame of an integrated safety supervision system with environment supervision, mine equipment monitoring and person / vehicle location management functions, and then carried out the HW\&SW design and development of the supervision substation device. Finally, a simulated test environment was built by the PC, USB-CAN interface card, the supervision substation device, ZigBee wireless sensor nodes and other modules; afterwards, the performance of the system structure and main functions of the supervision substation were validated. A photo of the test scene is shown in Figure 9.

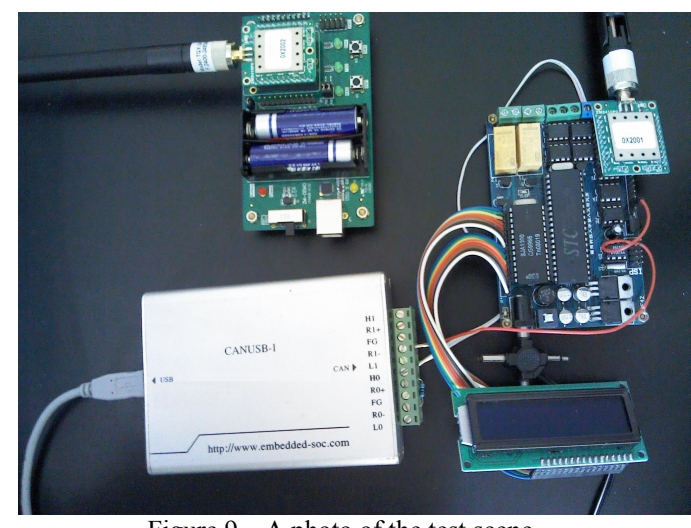

Figure 9. A photo of the test scene

\section{ACKNOWLEDGMENT}

This work was supported by the Planned Science and Technology Project of Hunan Province of China, under grant number: 2013FJ4046 and 2013FJ4048. Authors hereby express their thanks. 


\section{REFERENCES}

[1] ZHANG Guo Sheng, LIN An Dong. "Development Historical and Tendency of Mine Monitor System", Coal Technology, 2009.2, pp:8-9

[2] YU Xiu Wu. Study on Key Technologies of Wireless Sensor Networks in Mine Underground Safety Intelligent Monitorin, Wuhan: Wuhan University of Technology, 2013.

[3] LIU Yang. Design and Implementation of Environment Monitoring and Personnel Positioning System for Underground Coal Mine Based on Internet of Things, Beijing: Beijing Jiaotong University, 2011.

[4] Giron-Sierra J M, Insaurralde C, Seminario M. "CANbus-based Distributed Fuel System with Smart Components" ,IEEE Transactions on Aerospace and Electronic Systems, 2008, 44(3) ,pp: 897-912.

[5] Robert B G. CAN Specification V2.0 [EB/OL].
[E Http://www.semiconductors.bosch.de/pdf/can2spec.pdf, 2010-04-12.

[6] YI Li Qi. Design of Underground Mine Position System Based on IEEE802.15.4/ZigBee, Beijing: Beijing University of Posts and Telecommunications, 2010.

[7] YANG Chun hua. Research of wireless network coordinator based on ZigBee technology, Chengdu: Southwest Petroleum University, 2011.

[8] XU Jian, YANG Shan Shan. "The design of ZigBee coordinator node based on CC2530", Internet of Things Technologyies, 2012.2(5), pp: 55- 57 